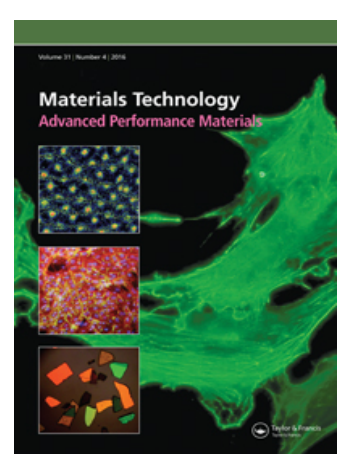

Materials Technology

Advanced Performance Materials

\title{
Effect of DOPA and dopamine coupling on protein loading of hydroxyapatite
}

\section{Ozhukil Kollath, S. Mullens, J. Luyten, K. Traina \& R. Cloots}

To cite this article: V. Ozhukil Kollath, S. Mullens, J. Luyten, K. Traina \& R. Cloots (2016) Effect of DOPA and dopamine coupling on protein loading of hydroxyapatite, Materials Technology, 31:4, 241-245, DOI: 10.1179/1753555715Y.0000000048

To link to this article: https://doi.org/10.1179/1753555715Y.0000000048

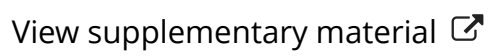

\section{曲 Published online: 11 Mar 2016.}

Submit your article to this journal ๘

Џ Article views: 157

View Crossmark data ¿

Citing articles: 1 View citing articles $4 \pi$ 


\title{
Effect of DOPA and dopamine coupling on protein loading of hydroxyapatite
}

\author{
V. Ozhukil Kollath ${ }^{*_{1,2,3}}$, S. Mullens ${ }^{2}$, J. Luyten ${ }^{2,4}$, K. Traina ${ }^{1,5}$ and R. Cloots ${ }^{*_{1}}$
}

\begin{abstract}
Hydroxyapatite $(\mathrm{HA})$ is a promising carrier material for oral delivery of biomolecules such as proteins and drugs. Ways to increase the loading of such molecules on HA will lead to better nanomedicine. This study reports the surface functionalisation of HA particles using the mussel inspired molecules dopamine (DA) and 3,4-dihydroxy-L-phenylalanine (DOPA), in order to increase protein loading. The adsorption mechanisms are discussed based on the adsorption isotherms, zeta potential, thermal analysis and theoretical models. Results show that DA functionalisation enhanced the loading, while DOPA functionalisation was ineffective.
\end{abstract}

Keywords: Hydroxyapatite, Protein, Dopamine, Adsorption isotherm, Oral delivery

\section{Introduction}

Oral delivery of therapeutic antigens (e.g. proteins, peptides) using nano- or micrometre sized carriers is an alternative for parenteral administration methods, which leads to enhanced patient convenience and compliance. ${ }^{1,2}$ Carrier mediated antigen delivery has shown enhanced bioavailability as compared to soluble antigens. ${ }^{3-5}$ The physicochemical properties of the carrier material play a major role in determining the cellular uptake of the administered antigen. This makes some of the inorganic carriers like calcium phosphate or mesoporous silica appealing due to their biocompatibility and surface modification possibilities. ${ }^{6-9}$ However, inorganic carriers used as carriers are still in the preclinical stage, and extensive research is necessary to assess their potential, as compared to the performance of polymeric carriers. ${ }^{10}$ Enhanced protein loading on the inorganic carrier is one such property to be studied carefully, which deals with the surface properties of the carrier material. Understanding the interaction mechanism and thus controlling the protein adsorption and release will also contribute to the bone implant materials development. ${ }^{11}$

The complex process of proteins adsorption onto solid surfaces is governed by forces like electrostatic, van der Waals and hydrophobic interactions. ${ }^{12}$ Various advanced physical and chemical surface engineering methods are reported in order to increase the protein adsorption on inorganic materials, to make them conducive for various applications like protein delivery, protein purification, waste water treatment, etc. ${ }^{13-17}$ A common platform for surface functionalisation with catechol containing molecules [e.g. 3,4-dihydroxy-L-phenylalanine (DOPA), dopamine (DA), norepinephrine (NA)] was reported on a variety of materials, including metal oxides,

${ }^{1}$ GREEnMat, Department of Chemistry, University of Liège, B6a SartTilman, Liège 4000, Belgium

${ }^{2}$ Sustainable Materials Management, Flemish Institute for Technological Research (VITO), Boeretang 200, Mol 2400, Belgium

${ }^{3}$ Current address: Faculty of Science, Laboratory of Anelasticity and Biomaterials, Univ. Estadual Paulista, Bauru, SP, 17033-360, Brazil ${ }^{4}$ Department of Metallurgy and Materials Engineering, Katholieke Universiteit Leuven, Heverlee 3001, Belgium

${ }^{5}$ Current address: Galephar S.A., Marche enFamenne 6900, Belgium

${ }^{*}$ Corresponding authors, emails vozhukil@alumni.ulg.ac.be (V. Ozhukil Kollath); rcloots@ulg.ac.be (R. Cloots) polymers and glass materials. ${ }^{18,19}$ Oxidative polymerisation of DA, a structural analogue of DOPA, was reported by Herlinger et al..$^{20}$ and has been exploited in several applications including underwater adhesives, non-fouling coatings, sensors and biomedical systems..$^{18,21,22}$ Nevertheless, reports of their use for inorganic carrier functionalisation are limited..$^{23,24}$ This study aims at increasing the protein loading on hydroxyapatite (HA) particles using DOPA and DA as linkers and improving the knowledge on the protein interaction.

Although oxidative polymerisation of these monomers has been reported, the exact mechanism is yet to be elucidated. ${ }^{25-27}$ Despite the use of these molecules in several applications including underwater adhesives, non-fouling coatings, sensors and biomedical systems, ${ }^{18,21,22,28}$ their use as a linker for protein adsorption on inorganic materials (e.g. calcium phosphate) is still underexplored. ${ }^{23,24}$ Researchers have tried to understand the protein adsorption on such materials by controlling the electrostatic and hydrophobic interactions. ${ }^{29,30}$

The choice of carrier material was made because of HA's known biocompatibility. ${ }^{9} 31,32$ Bovine serum albumin (BSA) was used as model protein in this study. Hydroxyapatite powder samples were functionalised with DOPA and DA solutions at $\mathrm{pH}$ 8.5. The influence of the functionalisations on the BSA adsorption isotherms (at pH 7.1) was analysed. The adsorption isotherms were fitted to Langmuir and Freundlich adsorption models. Further characterisation comprised zeta potential measurements, X-ray photon spectroscopy (XPS), Raman spectroscopy and thermogravimetric analysis online coupled with mass spectrometry (TGA-MS).

\section{Experimental}

Hydroxyapatite powder was purchased from Merck (product no. 1.02196; Darmstadt, Germany). Bovine serum albumin, Bradford reagent, Tris(hydroxymethyl)aminomethane (TRIS), DOPA and dopamine hydrochloride (DA) were purchased from Sigma-Aldrich. Highly pure water (Elix, Millipore) was used as solvent unless otherwise specified. All experiments were performed at room temperature.

For the DOPA and DA functionalisation, an aqueous solution of $2 \mathrm{mg} \mathrm{mL}^{-1}$ was prepared in $0.1 \mathrm{M}$ TRIS-HCl buffer 


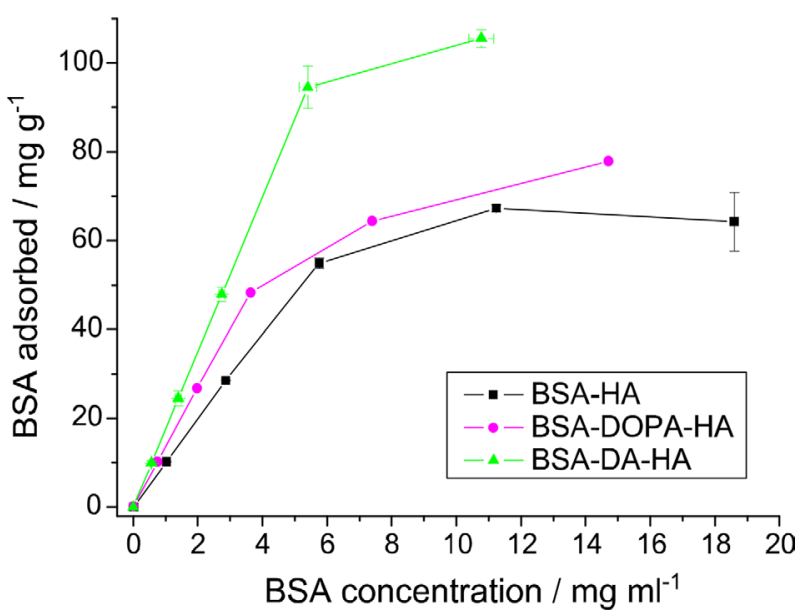

1 Bovine serum albumin adsorption isotherms of bare and functionalised HA

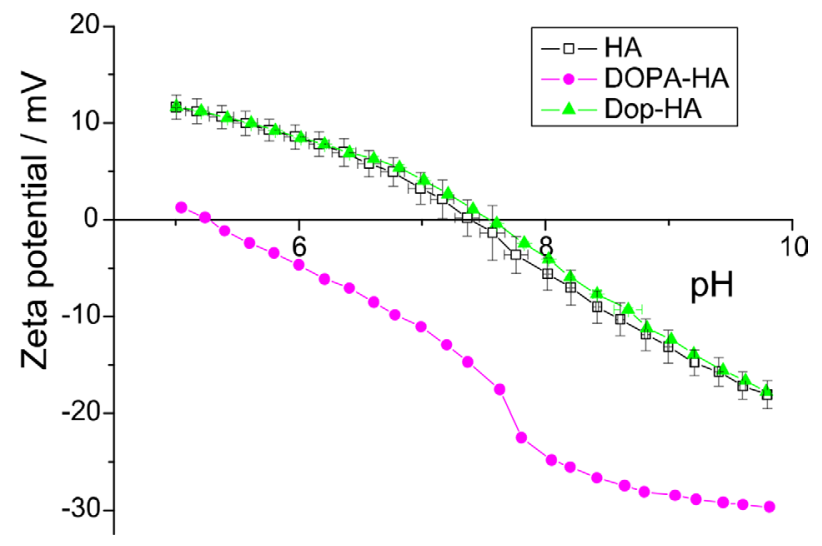

2 Zeta potential titration curves of HA, DOPA-HA and DA-HA

vpH 8.5). Two millilitres of these solutions were mixed with $0.2 \mathrm{~g}$ HA. These suspensions were stirred magnetically for $2 \mathrm{~h}$. After stirring, all the suspensions were centrifuged at $4000 \mathrm{rev} \mathrm{min}{ }^{-1}$ for $10 \mathrm{~min}$ (Centrifuge 5810, Eppendorf) in polypropylene centrifuge tubes. The supernatants were decanted, and $2 \mathrm{~mL}$ Milli-Q water was added to each tube to disperse the powder with the aid of a vortex mixer to wash off any loosely bound molecules. The tubes were centrifuged

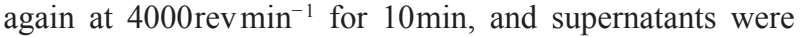
decanted. The residual paste in the tubes was dried at $40^{\circ} \mathrm{C}$ during $48 \mathrm{~h}$ in a drying oven, before characterisation.

Both the bare and functionalised HA powders were treated equally for the protein adsorption, and $\mathrm{pH}$ was kept equal to 7.1 in all cases. The $\mathrm{pH}$ of suspensions was adjusted to 7.1 for functionalised powders by adding $0.01 \mathrm{M} \mathrm{HCl}$. For BSA adsorption, a $2 \mathrm{~h}$ adsorption time was selected from experience. ${ }^{15}$ The adsorption experiments were performed by magnetically stirring a $10 \%(\mathrm{w} / \mathrm{v})$ suspension of HA powder in BSA solutions $(2 \mathrm{~mL})$ of varying concentrations (from 0 to $20 \mathrm{mg} \mathrm{mL}^{-1}$ ). Afterwards, the solution was centrifuged (Centrifuge 5810, Eppendorf AG, Germany) at 4000 rev $\mathrm{min}^{-1}$ for $6 \mathrm{~min}$ (in swing bucket rotor) and then at $11000 \mathrm{rev} \mathrm{min}^{-1}$ for $15 \mathrm{~min}$ (in fixed angle rotor). The residual paste of the suspension was then dried in air during $24 \mathrm{~h}$. Bradford assay was used to quantify the remaining BSA in the supernatant. The total amount of BSA adsorbed on the powder was obtained from the difference between initial and final (supernatant) concentrations of BSA in the solution. All adsorption measurements are represented as average values of at least duplicate experiments.

Thermogravimetric analyses (TGA) were performed (STA 449C, Netzsch) coupled to an online mass spectrometer (OmniStar GSD 301 O2, Pfeiffer Vacuum) in dry air atmosphere. Differential of the TG spectra provides the temperature of each weight loss events. Zeta potential measurements (Zetaprobe Analyzer, Colloidal Dynamics) were performed in the $\mathrm{pH}$ range of 5-10, and the titrations were performed using $\mathrm{HNO}_{3}(0.1 \mathrm{M})$ and $\mathrm{NaOH}(0.1 \mathrm{M})$. The Raman spectrum was acquired using Dilor XY in combination with and Olympus microscope (objective, $\times 50$ ). Green laser of $514 \mathrm{~nm}$ was used at $25 \mathrm{~mW}$ for 60 s during each acquisition. Elemental composition was analysed using XPS, and atomic percentages were calculated using the software provided by the manufacturer (Thetaprobe, Thermoelectron Corporation).

\section{Results and discussion}

Figure 1 shows the influence of the DOPA and DA functionalisation on the BSA adsorption isotherms. The maximum adsorption of BSA on DA-HA increased by $63 \%$ as compared to the bare HA. On the contrary, the DOPA functionalisation did not significantly change the BSA isotherm. The zeta potential measurements as a function of the $\mathrm{pH}$ (Fig. 2) showed the changes in surface charge and isoelectric point (IEP) of HA powder after functionalisation. No statistically relevant shift in zeta potential is measured after DA functionalisation (IEP of 7.5 for DA-HA as compared to 7.3 for the bare HA powder). After DOPA functionalisation, the zeta potential curve is shifted towards more negative zeta potential values, and the IEP is lowered to acidic $\mathrm{pH}$ (5.3).

It is known from the literature that, in alkaline $\mathrm{pH}, \mathrm{DA}$ will oxidise to quinone structure and subsequently polymerise to form polydopamine (PDA) (Scheme S1a; Supplementary material 1). ${ }^{18}$ Apart from the brownish colour change of the powder, Raman spectroscopy indicated the presence of PDA with characteristic broad peaks at 1604 and $1412 \mathrm{~cm}^{-1}$ (Fig. S1; Supplementary material 1). ${ }^{33,34}$ Preferentially, the catechol groups link with the hydroxyl and calcium ions on the HA surface. The final PDA structure contains no accessible functional groups, which can dissociate and change the zeta potential. The shift in IEP of DOPA-HA to the acidic $\mathrm{pH}$ is indicative of the presence of carboxylic functional groups on the particle surface. Assuming the catechol groups are interacting with the HA surface, the total surface charge is originating from the carboxyl groups of the dihydroxy-indole monomers (Scheme S1b; Supplementary material 1). Within the $\mathrm{pH}$ range of 3-8, the carboxyl group is deprotonated (Table S1; Supplementary material 1). ${ }^{35}$ Hence, the shift in zeta potential towards negative values (Fig. 2) indicates that the carboxylate group is electrostatically active on the surface of DOPA functionalised HA.

The TGA-MS (Fig. 3) was used to quantitatively measure the amount of DA and DOPA adsorbed on the HA powder. After DA functionalisation, the TGA curve shows an additional weight loss in the temperature range from 200 to $520^{\circ} \mathrm{C}$, with differential thermogravimetry (DTG) maxima at 300 and $424^{\circ} \mathrm{C}$. Online $\mathrm{MS}$ detected the release of $\mathrm{CO}_{2}(\mathrm{~m} / \mathrm{z}=44)$ and $\mathrm{H}_{2} \mathrm{O}(\mathrm{m} / \mathrm{z}=18)$ in the temperature range from 180 to $520^{\circ} \mathrm{C}$, assigned to the oxidation of the DA. A similar TGA profile was observed for DOPA-HA with DTG maxima at 314, 420 and $434^{\circ} \mathrm{C}$, assigned to the DOPA oxidation. The weight losses attributed to the oxidation are used to calculate the 

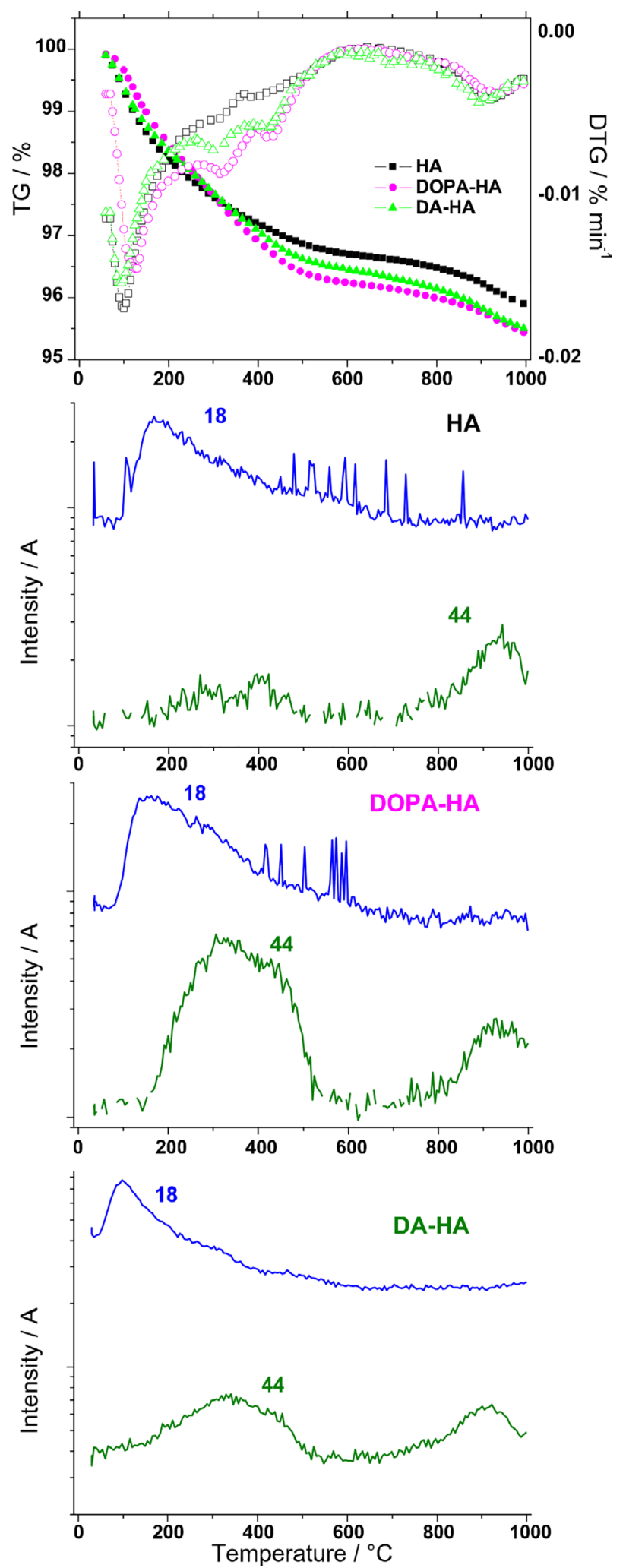

3 Thermogravimetric/DTG and mass spectra of bare and functionalised HA powders: curves with closed symbols represent TG, and open symbols represent DTG spectra respectively; mass spectra represent $\mathrm{H}_{2} \mathrm{O}(m / z=18)$ and $\mathrm{CO}_{2}(m / z=44)$ release during process

amount of DA and DOPA (Table S2; Supplementary material 1): $3.7 \times 10^{-5} \mathrm{molg}^{-1}$ powder was calculated for the DOPA functionalisation compared to $3.2 \times 10^{-5} \mathrm{~mol} \mathrm{~g}^{-1}$ powder for the DA functionalisation. Additionally to the quantitative analysis by TGA-MS, XPS was performed to measure the surface elemental composition (Table S3; Supplementary material 1). For DA-HA, 0.6at.\% nitrogen was measured. This result reinforces the idea that functionalisation occurred.

The combined characterisation from zeta potential measurements, Raman spectroscopy and TG offers us clues to the adsorption mechanism of BSA onto the functionalised HA. As the zeta potential as function of the $\mathrm{pH}$ does not change after DA functionalisation, it can be concluded that the total Coulomb interaction between protein and surface remains unchanged as compared with bare HA. However, the presence of PDA on the surface offers the possibility for covalent linking by a Michael addition or Schiff base reaction mechanism with the amine groups of BSA. ${ }^{18,36}$ Possible sites for Michael and Schiff base reactions are shown in Scheme S1 (Supplementary material 1). Furthermore, the decrease in hydrophilicity of the surface after PDA functionalisation could be contributed to the increase in BSA adsorption. ${ }^{18,37}$ Despite the increase in the repulsive electrostatic interaction after DOPA functionalisation, the BSA adsorption isotherm is similar to that of bare HA. Apart from electrostatic interactions, DOPA molecules on the HA surface can interact with BSA via Michael addition as reported earlier. ${ }^{38}$ Thus, the adsorption mechanism between BSA and DOPA-HA powder is a combination of electrostatic and covalent interactions.

Indications of this stronger bonding of BSA with polymerised DA can also be found in the TGA profile. Figure 4 compares the weight loss as function of the temperature for BSA adsorbed on HA and on DA-HA. The thermal decomposition of BSA adsorbed on HA is a two-step process, with DTG maxima at 323 and $450{ }^{\circ} \mathrm{C} .{ }^{39}$ The first weight loss is assigned to the oxidation and thermal decomposition of adsorbed BSA with the release of $\mathrm{CO}_{2}(m / z=44), \mathrm{H}_{2} \mathrm{O}(\mathrm{m} / z=18)$, $\mathrm{NO}$ and/or amines $(m / z=30)$ and traces of aromatic compounds $(m / z=77)$ (data not shown). The weight loss with DTG maximum at $450^{\circ} \mathrm{C}$ can be attributed to the combustion of the char with formation of only $\mathrm{CO}_{2}$ and $\mathrm{NO}(\mathrm{m} / z=30)$. In case of DA-HA, the second DTG maximum is shifted to $470^{\circ} \mathrm{C}$. This shift of $\sim 20^{\circ} \mathrm{C}$ might indicate a stronger interaction of BSA after functionalisation.

The differences in adsorption mechanisms after DA and DOPA functionalisation also emerge from fitting the adsorption isotherms to the Langmuir and Freundlich models (Table S4). The adsorption isotherms of bare HA and DOPA-HA are described more closely by the Langmuir model. After DA functionalisation, the Freundlich model is in better agreement

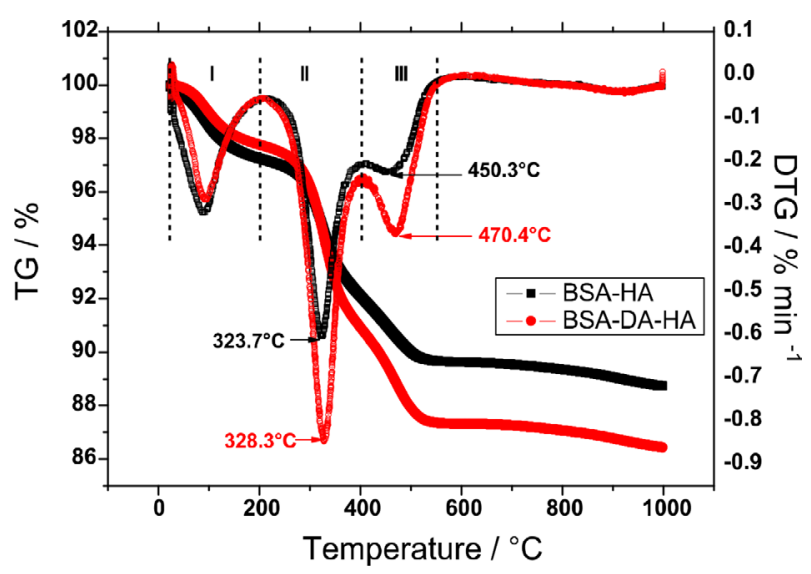

4 Thermogravimetric and DTG spectra of BSA on bare and DA functionalised HA: curves with closed symbols represent TG, and open symbols represent DTG spectra respectively 
with the experimental data. These data indicate the formation of BSA multilayer on DA-HA.

When normalising the maximum BSA adsorption by the surface area (Table S4) and comparing it with calculated values of full surface coverage, information is obtained on the conformation of the BSA on the surface. It has been reported that BSA can adsorb either in a side-on or end-on mode, with full surface coverage amounts of 172 and $309 \mathrm{ng} \mathrm{cm}^{-2}$ respectively in case of random adsorption. ${ }^{40}$ This random adsorption model accounts $54.7 \%$ of jamming limit and hence a loosely packed layer. In case of BSA adsorption on HA or DOPA-HA, the maximum adsorption amounts are below the calculated monolayer amounts for side-on adsorption. But in case of BSA adsorbed on DA-HA, the maximum BSA corresponds to the random side-on monolayer threshold.

\section{Conclusions}

In summary, the interaction of BSA with DOPA and DA functionalised HA was studied. Protein loading on DA-HA powder increased by $63 \%$ as compared to the bare HA powder. The wide range of characterisation tools and the comparison with theoretical models yielded more insight in the complex adsorption mechanisms involved. However, more research is needed to clarify all aspects of the polymerisation mechanism of DA and DOPA. Future work will focus on comparing the current method with other linker molecules to enhance the BSA adsorption as well as the protein release properties of the developed materials.

\section{Acknowledgements}

The authors gratefully acknowledge I. K. Baert and Professor I. Vandendael (SURF, VrijeUniversiteit Brussel) for Raman spectroscopy, and Dr B. G. De Geest, Professor J. P. Remon (Laboratory of Pharmaceutical Technology, Ghent University) and Dr V. Meynen (Laboratory of adsorption and catalysis, University of Antwerp) for fruitful discussions. VOK wish to thank VITO and University of Liège for financial assistance and M. Sharma for proofreading the manuscript.

\section{Supplementary material}

The supplemental material for this paper is available at http:// dx.doi.10.1179/1753555715Y.0000000048

\section{References}

1. A. Grenha: 'Systemic delivery of biopharmaceuticals: Parenteral forever?', J. Pharm. Bioallied Sci., 2012, 4, 95.

2. M. L. Hoffman-Terry, H. S. Fraimow, T. R. Fox, B. G. Swift and J. E. Wolf: 'Adverse effects of outpatient parenteral antibiotic therapy', Am. J. Med., 1999, 106, 44

3. M. Singh and D. O'Hagan: 'Advances in vaccine adjuvants', Nat. Biotechnol., 1999, 17, 1075-1081.

4. S. De Koker, B. N. Lambrecht, M. A. Willart, Y. van Kooyk, J. Grooten, C. Vervaet, J. P. Remon and B. G. De Geest: 'Designing polymeric particles for antigen delivery', Chem. Soc. Rev., 2011, 40, 320-339, M. Dierendonck, S. De Koker, C. Cuvelier, J. Grooten, C. Vervaet, J.P. Remon and B.G. De Geest: Facile two-step synthesis of porous antigenloaded degradable polyelectrolyte microspheres Angew. Chem. Int. Ed., 2010, 49, 8620-8624.

5. L. J. De Cock, S. De Koker, B. G. De Geest, J. Grooten, C. Vervaet, J. P. Remon, G. B. Sukhorukov and M. N. Antipina: 'Polymeric multilayer capsules in drug delivery', Angew. Chem. Int. Ed., 2010, 49, 6954-6973.
6. E. V. Giger, J. Puigmartí-Luis, R. Schlatter, B. Castagner, P. S. Dittrich and J. -C. Leroux: 'Gene delivery with bisphosphonate-stabilized calcium phosphate nanoparticles', J. Controlled Release, 2011, 150, 87-93.

7. M. Vallet-Regí, F. Balas and D. Arcos: 'Mesoporous materials for drug delivery', Angew. Chem. Int. Ed., 2007, 46, 7548-7558.

8. G. Bhakta, S. Mitra and A. Maitra: 'DNA encapsulated magnesium and manganous phosphate nanoparticles: potential non-viral vectors for gene delivery', Biomaterials, 2005, 26, 2157-2163.

9. V. V. Sokolova, I. Radtke, R. Heumann and M. Epple: 'Effective transfection of cells with multi-shell calcium phosphate-DNA nanoparticles', Biomaterials, 2006, 27, 3147-3153.

10. Z. P. Xu, Q. H. Zeng, G. Q. Lu and A. B. Yu: 'Inorganic nanoparticles as carriers for efficient cellular delivery', Chem. Eng. Sci., 2006, 61, 1027-1040.

11. S. Tarafder, S. Banerjee, A. Bandyopadhyay and S. Bose: 'Electrically polarized biphasic calcium phosphates: adsorption and release of bovine serum albumin', Langmuir, 2010, 26, 16625-16629.

12. M. Rabe, D. Verdes and S. Seeger: 'Understanding protein adsorption phenomena at solid surfaces', Adv. Colloid Interface Sci., 2011, 162, 87-106.

13. K. Cai, M. Frant, J. Bossert, G. Hildebrand, K. Liefeith and K. D. Jandt: 'Surface functionalized titanium thin films: Zeta-potential, protein adsorption and cell proliferation', Colloids Surf. B, 2006, 50B, 1-8.

14. D. Kozlova, S. Chernousova, T. Knuschke, J. Buer, A. M. Westendorf and M. Epple: 'Cell targeting by antibodyfunctionalized calcium phosphate nanoparticles', J. Mater. Chem., 2011, 22, 396-404.

15. V. Ozhukil Kollath, B. G. Geest, S. Mullens, S. De Koker, J. Luyten, R. Persoons, K. Traina, J. P. Remon and R. Cloots: 'Systematic processing of $\beta$-tricalcium phosphate for efficient protein loading and in vitro analysis of antigen uptake', Adv. Eng. Mater., 2013, 15, 295-301.

16. V. Ozhukil Kollath, S. Put, S. Mullens, A. Vanhulsel, J. Luyten, K. Traina and R. Cloots: 'Atmospheric pressure plasma as an activation step for improving protein adsorption on hydroxyapatite powder', Plasma Process Polym., 2015, 12, 594-601, DOI: 10.1002/ppap.201400092

17. V. Ozhukil Kollath, F. van den Broeck, K. Fehér, J. C. Martins, J. Luyten, K. Traina, S. Mullens and R. Cloots: 'A modular approach to study protein adsorption on surface modified hydroxyapatite', Chem.-Eur. J., 2015, DOI: 10.1002/chem.201500223. Chemistry A, to be published.

18. H. Lee, S. M. Dellatore, W. M. Miller and P. B. Messersmith: 'Musselinspired surface chemistry for multifunctional coatings Science', 2007, 318, 426-430, B. P. Lee, P. B. Messersmith, J. N. Israelachvili and J. H. Waite: Mussel-inspired adhesives and coatings Annu. Rev. Mater. Res., 2011, 41, 99-132.

19. S. M. Kang, J. Rho, I. S. Choi, P. B. Messersmith and H. Lee: 'Norepinephrine: material-independent, multifunctional surface modification reagent', J. Am. Chem. Soc., 2009, 131, 13224-13225.

20. E. Herlinger, R. F. Jameson and W. Linert: 'Spontaneous autoxidation of dopamine', J. Chem. Soc. Perkin Trans., 1995, 2, 259-263.

21. Q. Ye, F. Zhou and W. Liu: 'Bioinspired catecholic chemistry for surface modification', Chem. Soc. Rev., 2011, 40, 4244-4258.

22. J. Sedó, J. Saiz-Poseu, F. Busqué and D. Ruiz-Molina: 'Catechol-based biomimetic functional materials', Adv. Mater., 2013, 25, 653-701.

23. Y. Lee, S. H. Lee, J. S. Kim, A. Maruyama, X. Chen and T. G. Park: 'Controlled synthesis of PEI-coated gold nanoparticles using reductive catechol chemistry for siRNA delivery', J. Controlled Release, 2011, 155, 3-10.

24. G. Marcelo, A. Muñoz-Bonilla, J. Rodríguez-Hernández and M. Fernández-García: 'Hybrid materials achieved by polypeptide grafted magnetite nanoparticles through a dopamine biomimetic surface anchored initiator', Polym. Chem., 2013, 4, 558-567.

25. M. D'Ischia, A. Napolitano, A. Pezzella, P. Meredith and T. Sarna: 'Chemical and structural diversity in eumelanins: Unexplored biooptoelectronic materials', Angew. Chem. Int. Ed., 2009, 48, 3914-3921.

26. D. R. Dreyer, D. J. Miller, B. D. Freeman, D. R. Paul and C. W. Bielawski: 'Elucidating the structure of poly(dopamine)', Langmuir, 2012, 28, 6428-6435.

27. S. Hong, Y. S. Na, S. Choi, I. T. Song, W. Y. Kim and H. Lee: 'Noncovalent self-assembly and covalent polymerization co-contribute to polydopamine formation', Adv. Funct. Mater., 2012, 22, 4711-4717.

28. J. I. Lim, J. H. Kim and H. -K. Park: 'The adhesive properties of prepolymerized allyl 2-cyanoacrylate/poly L-3,4-dihydroxyphenylalanine for use as bioglue', Mater. Lett., 2012, 81, 251-253.

29. F. Meder, T. Daberkow, L. Treccani, M. Wilhelm, M. Schowalter, A. Rosenauer, L. Mädler and K. Rezwan: 'Controlling protein-particle adsorption by surface tailoring colloidal alumina particles with sulfonate groups', Acta Biomater., 2012, 8, 1221-1229.

30. S. Dasgupta, A. Bandyopadhyay and S. Bose: 'Reverse micelle-mediated syntehsis of calcium phosphate nanocarriers for controlled release of bovine serum albumin', Acta Biomater., 2009, 5, 3112-3121. 
31. H. Fu, M. N. Rahaman, D. E. Day and R. F. Brown: 'Hollow hydroxyapatite microspheres as a device for controlled delivery of proteins', J. Mater. Sci.: Mater. Med., 2011, 22, 579-591.

32. R. Ramachandran, W. Paul and C. P. Sharma: 'Synthesis and characterization of PEGylated calcium phosphate nanoparticles for oral insulin delivery', J. Biomed. Mater. Res. B, 2009, 88B, 41-48.

33. N. G. Rim, S. J. Kim, Y. M. Shin, I. Jun, D. W. Lim, J. H. Park and H. Shin: 'Mussel-inspired surface modification of poly(L-lactide) electrospun fibers for modulation of osteogenic differentiation of human mesenchymal stem cells', Colloids Surf. B, 2012, 91B, 189-197.

34. J. Ryu, S. H. Ku, H. Lee and C. B. Park: Adv. Funct. Mater., 2010, 20 2132-2139.

35. X. Chen, J. Xie, C. Li, Z. Hu and X. Chen: 'Investigation of the factors that induce analyte peak splitting in capillary electrophoresis', J. Sep. Sci., 2007, 27, 1005-1010.
36. C. Cheng, S. Nie, S. Li, H. Peng, H. Yang, L. Ma, S. Sun and C. Zhao: 'Biopolymer functionalized reduced graphene oxide with enhanced biocompatibility via mussel inspired coatings/anchors', J. Mater. Chem. $B, 2013, \mathbf{1 B}, 265-275$

37. J. -Y. Yoon, J. -H. Kim and W. -S. Kim: 'Interpretation of protein adsorption phenomena onto functional microspheres', Colloids Surf. B, 1998, 12B, 15-22.

38. M. Yu, J. Hwang and T. H. Deming: 'Role of L-3,4-dihydroxyphenylalanine in mussel adhesive proteins', J. Am. Chem. Soc., 1999, 121, 5825-5826.

39. T. -Y. Liu, S. -Y. Chen, D. -M. Liu and S. -C. Liou: 'On the study of BSAloaded calcium-deficient hydroxyapatite nano-carriers for controlled drug delivery', J. Controlled Release, 2005, 107, 112-121.

40. B. Mueller, M. Zacharias and K. Rezwan: 'Bovine serum albumin and lysozyme adsorption on calcium phosphate particles', Adv. Eng. Mater., 2010, 12, B53-B61. 